\title{
Staging Uveal Melanoma with Whole-Body Positron-Emission Tomography/Computed Tomography and Abdominal Ultrasound: Low Incidence of Metastatic Disease, High Incidence of Second Primary Cancers
}

\author{
$\underline{\text { Victoria M. L. Cohen, },{ }^{1,2} \text { Efthymia Pavlidou, }},{ }^{1}$ Joanna DaCosta,,${ }^{1}$ Amit K. Arora, ${ }^{1}$ Teressa Szyszko, ${ }^{3} \underline{\text { Mandeep }}$ \\ S. Sagoo, ${ }^{1,2}$ and Peter Szlosarek ${ }^{4}$
}

Address for correspondence: Miss. Victoria M.L. Cohen, Cohen, Moorfields Eye Hospital, 162 City Road, EC1V 2PD,

London, UK. E-mail ku.shn.sdleifroom@nehoc.airotciv

Copyright : ( 2018 Middle East African Journal of Ophthalmology

This is an open access journal, and articles are distributed under the terms of the Creative Commons Attribution-

NonCommercial-ShareAlike 4.0 License, which allows others to remix, tweak, and build upon the work non-commercially, as

long as appropriate credit is given and the new creations are licensed under the identical terms.

\section{Introduction}

Uveal melanoma, as the most common primary intraocular tumor in adults, has a reported incidence of 5-7 cases per million of the population. [1] According to long-term survival studies, metastases develop in up to $50 \%$ of cases within 15 years of diagnosis.[2] Metastatic choroidal melanoma generally presents in the liver.[2,3,4] In most series, hepatic involvement is reported in up to $95 \%$ of patients, while $50 \%$ are noted to have coexisting extrahepatic metastases (lung, bone, and skin).[5] Historically, abdominal ultrasound has been used to stage patients with uveal melanoma because metastatic disease is usually first found in the liver.

Whole-body positron-emission tomography/computed tomography (PET/CT) has been used for staging of systemic lymphoma, cutaneous melanoma, and gastrointestinal malignancies. PET was developed from the knowledge that cancer cells display-deregulated energetics that is linked to increased glucose consumption.[6] PET/computed tomography scanning uses radioactive glucose [18-fluoro-2-deoxyglucose (FDG)] that accumulates within neoplastic lesions that contain metabolically active cells. [?] The FDG injected patient is imaged on a whole-body PET scanner, the computed tomography portion of a PET/CT provides anatomic information about the location, size, and shape of tumors. Ultimately, the anatomic and metabolic findings are combined to provide information on function and location.

Whole-body PET/CT imaging is gaining worldwide acceptance in the initial staging, screening and management of patients with cancer.[ $\left.{ }^{[, 9}\right]$

The objective of this study was to report the value of staging uveal melanoma with FDGPET/CT and abdominal ultrasound. 
From January 2012, patients with primary uveal malignant melanoma over $4 \mathrm{~mm}$ in thickness were staged with 18-FDG) whole-body PET/CT (Phillips Gemini TF LS064) and abdominal ultrasound. $4 \mathrm{~mm}$ was selected as the cut off because this is the minimum resolution of the PET/CT scan. Tumors $<4 \mathrm{~mm}$ in thickness were staged with abdominal ultrasound only but not included in this study. The imaging findings of each modality were compared in a retrospective case note analysis. We recorded the incidence of metastatic uveal melanoma at diagnosis and other incidental findings detected using both imaging techniques. We recorded the size, location and the international tumor, node, and metastasis (TNM) stage of each primary uveal melanoma. If abnormalities were noted on PET/CT scan or abdominal ultrasound, patients were further investigated with CT and magnetic resonance imaging (MRI) scans (with contrast) or liver biopsy to confirm the presence of metastatic disease.

\section{Positron emission tomography/computed tomography imaging}

Patients were instructed to refrain from eating a carbohydrate-based dinner the previous night and to fast 4-6 h before injection of 18-fluoro-2-deoxyglucose (FDG). These measures diminish physiological glucose utilization and reduce insulin serum levels to baseline. The full body PET/CT scan began at the top of the head and ended at the bottom of the feet. The CT portion consisted of a multi-detector helical CT scanner. The FDG was injected via an intravenous line followed by $10 \mathrm{ml}$ of normal saline. The resolution of the PET scan was 4 $\mathrm{mm}$. The PET scan reconstructed the images and utilized the CT scan to correct for attenuation. Then workstation was used to fuse and display the PET and CT images and to produce the final image [Figure 1].

\section{Positron emission tomography/computed tomography image evaluation}

The PET/CT images were studied for the presence of lymph node and distant metastases and were based on qualitative analysis. Data were evaluated for areas of focally increased glucose uptake and by maximally standardized uptake values (SUV). Glucose uptake above levels of the surrounding tissue qualitatively and an uptake value above 2.5 SUV quantitatively indicated malignancy. Distant disease was confirmed via biopsy or additional imaging. This study was approved by the Institutional Review Board and adhered to the tenets of The Declaration of Helsinki.

\section{Results}

Between January 2012 and January 2014, 108 patients underwent staging for primary uveal melanoma with whole-body FDG PET/CT and abdominal ultrasound. There were 10 anterior uveal melanomas, of which 7 were located in the ciliary body, one had iridociliary involvement and there were two ring melanomas. Of the remaining 98 tumors, 83 were choroidal and 15 had ciliochoroidal involvement. The mean tumor height was $7.9 \mathrm{~mm}$ while the mean tumor diameter was $13.1 \mathrm{~mm}$ [Table 1].

\section{Using the collaborative ocular melanoma study group}

Classification, there were 5 small (4.6\%), 46 medium (42.6\%), and 57 large (52.7\%) tumors. The TNM classification $7^{\text {th }}$ edition of the American Joint Committee on Cancer (AJCC) Cancer Staging Manual 2009 was also used to categorize uveal melanoma [Table 1]. 
Three of 108 patients $(2.7 \%)$ were found to have distant metastatic disease at staging. Two had posterior choroidal melanoma and one had a ciliary body melanoma. Two of the 3 metastatic patients presented with large tumors based on the Collaborative Ocular Melanoma Study criteria and were treated with enucleation. The third case had a medium sized melanoma and was treated with plaque brachytherapy. All 3 patients had hepatic metastases, confirmed on biopsy in one case [Figure 2]. One of the three metastatic cases had additional extrahepatic widespread metastases in the lungs, lymph nodes, and bones seen on whole-body FDG PET/CT.

The hepatic findings using abdominal ultrasound and PET/CT were consistent in one patient. In 2 of 3 patients with metastatic disease, the hepatic findings using abdominal ultrasound and PET/CT differed. In the first case, the abdominal ultrasound misdiagnosed metastatic disease as hepatic cysts; however, FDG PET/CT revealed several foci of intense metabolic activity in both lobes of the liver. In the second case, PET/CT missed the presence of hepatic metastases as no metabolic activity was detected, however a hypodense hepatic lesion was identified on abdominal ultrasound. An abdominal CT scan with contrast confirmed the presence of an enlarging hepatic metastasis.

PET/CT identified second primary malignancies in 10 patients $(9.3 \%)$. The second primary cancers were found in the lung, breast, colon, thyroid, and adrenal gland. All patients with a second cancer were referred to the appropriate multidisciplinary team for specialist treatment at St Bartholomew's Cancer Centre. Incidental extrahepatic pathology was found in another 12 patients $(11.1 \%)$.

Coincidental benign liver findings, such as fatty liver, hepatic cysts or hemangiomata were seen in 20 patients (18.5\%) using abdominal ultrasound. Whereas, whole-body PET/CT detected benign liver pathology in only 4 patients (3.7\%) within the same cohort.

\section{Discussion}

The detection of metastatic uveal melanoma at diagnosis of the primary tumour is a rare event but has major prognostic implications. The median survival for stage IV disease in uveal melanoma is approximately 6-9 months with $80 \%$ of deaths occurring within the $1^{\text {st }}$ year of metastatic disease. $\left[{ }^{10}\right]$ The UK National Guidelines for the management of Uveal Melanoma, published in the European Journal of Cancer, recommend staging uveal melanoma patients. $\left[\frac{11}{}\right]$ Although, the detection of distant metastases may not necessarily influence the management of the primary uveal melanoma.[프 $]$ The authors suggested that further research is required to determine the choice imaging modality.[11]

There have been a few publications on the use of liver function tests and chest X-ray in staging patients with uveal melanoma.[11,12] It was concluded that these investigations have a high specificity but not a high sensitivity for the early detection of metastatic disease. Staging uveal melanoma patients with full-body PET/CT has been proposed to increase diagnostic accuracy.[13] PET/CT imaging has been used in the diagnosis, staging, and restaging of various cancers. $\left[{ }^{8,9,14}\right]$ The combination of metabolic and structural information provided by the PET/CT has improved the accuracy of tumor staging, detection of recurrences and has a significant impact on patient management.[눈 High sensitivity rates and the value of FDGPET for the detection of hepatic metastases from uveal melanoma have already been reported. $\left[\frac{9,13,15]}{}\right.$ Klingenstein et al. $\left[\frac{16}{6}\right]$ reported that PET/CT detected metastases from uveal melanoma in all of their patients. 
Ionizing imaging such as PET/CT is not recommended for surveillance of metastatic uveal melanoma due to the high radiation doses associated with this imaging modality.[11] In our study, we report the use of PET/CT as a staging tool only. All patients receive long-term surveillance every 6 months with non-ionizing imaging, namely abdominal MRI or ultrasound.

\section{Metastatic uveal melanoma}

In our series, only $3(2.7 \%)$ uveal melanoma patients were found to have metastases at the time of diagnosis, this is consistent with the overall incidence of metastatic disease at diagnosis reported by other investigators.[13] In our study, PET/CT identified hepatic metastases in 2 of 3 patients $(66.6 \%)$. In contrast, abdominal ultrasound identified hepatic abnormalities in all three patients. In two patients $(66.6 \%)$, the liver ultrasound accurately detected hepatic metastases, in the third case metastatic deposits were misdiagnosed as hepatic cysts. Staging with PET/CT allowed the detection of extra-hepatic metastases this is important as up to $75 \%$ of patients with metastatic uveal melanoma often have multiple sites of involvement, primarily lung and bone.[르 Early detection allows early intervention. Although success of treatment is limited, surgical intervention, targeted hepatic chemotherapy, localized radiotherapy, small molecule kinase inhibitors and immune system modulators such as PD1 and MEK inhibitors are being now all being used in the management of patients with metastatic melanoma. $[\underline{17,18}]$

\section{Second cancers}

Other extra-hepatic lesions found on PET/CT scanning included second primary malignancy in $9.3 \%$. Early detection of a second asymptomatic primary cancer can improve patient survival. $[1,19]$ Studies have found that detection of a second primary cancer following the diagnosis of an apparently unrelated cancer is not uncommon. $[1,20,21,22]$ In fact, cancer patients have approximately twice the risk of the development of an additional primary cancer than cancer-free patients of the same sex and age.[를 In our series, whole-body PET/CT was able to provide early detection of asymptomatic, unrelated second primary cancers in almost 1 in 10 patients with uveal melanoma. None of these patients reported symptoms that would have indicated a second primary cancer. Second primary cancers were found in the lung, breast, colon, thyroid and adrenal gland. The discovery of a second cancer was a somewhat unexpected, but important result. Hereditary BAP1 cancer predisposition syndrome provides a genetic explanation for the association of uveal melanoma with cutaneous melanoma, malignant pleural mesothelioma and renal cell carcinoma.[남] However, none of the second cancers found on our cohort were typical tumors seen in the described germline BAP1 mutation. Nevertheless, this is a rapidly developing field; cholangiocarcinoma and breast cancer may also be part of the BAP1 phenotype.[24] Other candidate genes are likely to be involved in addition to BAP 1 . This underscores the need to assess the whole patient following a diagnosis of uveal melanoma so that optimal patient care can be achieved in close collaboration with a medical oncologist.

\section{Imaging technique}

Performance of FDG-PET imaging is limited by numerous technical and methodological factors. First, it is more difficult to detect even metabolically active lesions if their size is $<1$ $\mathrm{cm}$. Second, FDG uptake reflects glucose metabolic flux, which is physiologically present in normal liver. Thus, the normal "mottled" background is another factor which decreases the sensitivity of liver lesion detection and the target to background ratio. Lesions that are quite 
large may not be detected if their metabolism matches that of the patient's liver. The last important limitation of PET/CT imaging is the respiratory motion that produces localization and reconstruction artefacts. Respiratory gating techniques, mainly used in chest pathologies, should possibly improve the results. Servois et al. $\left[{ }^{25}\right]$ proved that MRI is superior to PET alone in a small preoperative case series of uveal melanoma patients with hepatic metastases. Yet, they focused on hepatic involvement. They acknowledged that PET, as whole-body imaging modality, presents the advantage of also detecting extrahepatic metastases. The limitations of PET scan alone such as, the partial volume effect, physiologically high intrahepatic glucose metabolism, and artifacts by respiratory motion can be largely overcome by using combined PET/CT scan and adequate breathing techniques.[16]

Ultrasound is a noninvasive, simple to perform and a low cost technique, which provides detailed examination and in our series was superior to the PET/CT scan in detecting hepatic abnormalities and providing details of many other benign abnormalities. Ultrasound provides the immediate advantage of instant reporting. However, ultrasound technique is operator dependent and therefore the quality of the report can vary with the experience of the operator. In this study, ultrasound scans were performed by highly experienced radiologists our cancer institution.

In Summary, the incidence of metastatic uveal melanoma at the time of primary diagnosis remains low even for larger tumors. Benign hepatic disease is common and best detected with abdominal ultrasound, which provides a baseline description of the liver architecture and is useful for future screening. Full-body PET/CT allows detection of extra-hepatic disease; we report a second cancers detection rate of $9.3 \%$, which is highly relevant. Therefore, the dual imaging techniques complement each other in the early detection of metastatic disease and second malignancies in uveal melanoma. No cost benefit analysis was undertaken in this study and ultimately this might adversely affect the global uptake of PET/CT imaging for staging uveal melanoma. However, it is important to remember the high incidence of an asymptomatic, second primary malignancy in this group of patients.

\section{Conclusion}

Whole body PET/CT and abdominal ultrasound complement each other in the staging of uveal melanoma. Benign hepatic abnormalities found using ultrasound are common. A second asymptomatic primary malignancy was seen in almost $10 \%$ of patients with uveal melanoma.

\section{Financial support and sponsorship}

Nil.

\section{Conflicts of interest}

There are no conflicts of interest.

\section{References}

1. Hu DN, Yu G, McCormick SA, Finger PT. Population-based incidence of conjunctival melanoma in various races and ethnic groups and comparison with other melanomas. Am J Ophthalmol. 2008;145:418-23. [PubMed: 18191091]

2. Kujala E, Mäkitie T, Kivelä T. Very long-term prognosis of patients with malignant uveal melanoma. Invest Ophthalmol Vis Sci. 2003;44:4651-9.[PubMed: 14578381] 
3. Zimmerman LE. Metastatic disease from uveal melanomas. A review of current concepts with comments concerning future research and prevention. Trans Ophthalmol Soc U

K. 1980;100:34-54. [PubMed: 7022781]

4. Einhorn LH, Burgess MA, Gottlieb JA. Metastatic patterns of choroidal melanoma. Cancer. 1974;34:1001-4. [PubMed: 4424282]

5. Flaherty LE, Unger JM, Liu PY, Mertens WC, Sondak VK. Metastatic melanoma from intraocular primary tumors: The Southwest Oncology Group experience in phase II advanced melanoma clinical trials. Am J Clin Oncol. 1998;21:568-72. [PubMed: 9856657]

6. Kapoor V, McCook BM, Torok FS. An introduction to PET-CT imaging. Radiographics. 2004;24:523-43. [PubMed: 15026598]

7. Lindholm P, Minn H, Leskinen-Kallio S, Bergman J, Ruotsalainen U, Joensuu H, et al. Influence of the blood glucose concentration on FDG uptake in cancer - A PET study. J Nucl Med. 1993;34:1-6. [PubMed: 8418248]

8. Schöder H, Larson SM, Yeung HW. PET/CT in oncology: Integration into clinical management of lymphoma, melanoma, and gastrointestinal malignancies. J Nucl Med. 2004;45(Suppl 1):72S-81S. [PubMed: 14736838]

9. Freudenberg LS, Schueler AO, Beyer T, Antoch G, Kühl H, Bornfeld N, et al. Whole-body fluorine-18 fluordeoxyglucose positron emission tomography/computed tomography (FDGPET/CT) in staging of advanced uveal melanoma. Surv Ophthalmol. 2004;49:53740. [PubMed: 15325198]

10. Gragoudas ES, Egan KM, Seddon JM, Glynn RJ, Walsh SM, Finn SM, et al. Survival of patients with metastases from uveal melanoma. Ophthalmology. 1991;98:383-9. [PubMed: 2023760]

11. Nathan P, Cohen V, Coupland S, Curtis K, Damato B, Evans J, et al. Uveal melanoma UK national guidelines. Eur J Cancer. 2015;51:2404-12. [PubMed: 26278648]

12. Diener-West M, Reynolds SM, Agugliaro DJ, Caldwell R, Cumming K, Earle JD, et al. Screening for metastasis from choroidal melanoma: The Collaborative Ocular Melanoma Study Group Report 23. J Clin Oncol. 2004;22:2438-44. [PubMed: 15197206]

13. Finger PT, Kurli M, Reddy S, Tena LB, Pavlick AC. Whole body PET/CT for initial staging of choroidal melanoma. Br J Ophthalmol. 2005;89:1270-4.[PMCID:

PMC1772897] [PubMed: 16170114]

14. Finger PT, Kurli M, Wesley P, Tena L, Kerr KR, Pavlick A, et al. Whole body PET/CT imaging for detection of metastatic choroidal melanoma. Br J Ophthalmol. 2004;88:10957. [PMCID: PMC1772278] [PubMed: 15258035]

15. Francken AB, Fulham MJ, Millward MJ, Thompson JF. Detection of metastatic disease in patients with uveal melanoma using positron emission tomography. Eur J Surg Oncol. 2006;32:780-4. [PubMed: 16765562]

16. Klingenstein A, Haug AR, Nentwich MM, Tiling R, Schaller UC. Whole-body F-18fluoro-2-deoxyglucose positron emission tomography/computed tomography imaging in the follow-up of metastatic uveal melanoma. Melanoma Res. 2010;20:511-6. [PubMed: 20890225] 
17. Leyvraz S, Spataro V, Bauer J, Pampallona S, Salmon R, Dorval T, et al. Treatment of ocular melanoma metastatic to the liver by hepatic arterial chemotherapy. J Clin

Oncol. 1997;15:2589-95. [PubMed: 9215829]

18. Feldman ED, Pingpank JF, Alexander HR., Jr Regional treatment options for patients with ocular melanoma metastatic to the liver. Ann Surg Oncol. 2004;11:290-7.[PubMed: 14993024]

19. Choi JY, Lee KS, Kwon OJ, Shim YM, Baek CH, Park K, et al. Improved detection of second primary cancer using integrated $[18 \mathrm{~F}]$ fluorodeoxyglucose positron emission tomography and computed tomography for initial tumor staging. J Clin

Oncol. 2005;23:7654-9. [PubMed: 16234527]

20. van Leeuwen FE, Klokman WJ, Stovall M, Dahler EC, van't Veer MB, Noordijk EM, et al. Roles of radiation dose, chemotherapy, and hormonal factors in breast cancer following Hodgkin's disease. J Natl Cancer Inst. 2003;95:971-80. [PubMed: 12837833]

21. Obedian E, Fischer DB, Haffty BG. Second malignancies after treatment of early-stage breast cancer: Lumpectomy and radiation therapy versus mastectomy. J Clin Oncol. 2000;18:2406-12. [PubMed: 10856100]

22. Diener-West M, Reynolds SM, Agugliaro DJ, Caldwell R, Cumming K, Earle JD, et al. Second primary cancers after enrollment in the COMS trials for treatment of choroidal melanoma: COMS report no 25. Arch Ophthalmol. 2005;123:601-4. [PubMed: 15883277]

23. Dong C, Hemminki K. Second primary neoplasms in 633,964 cancer patients in Sweden, 1958-1996. Int J Cancer. 2001;93:155-61. [PubMed: 11410860]

24. Pilarski R, Cebulla CM, Massengill JB, Rai K, Rich T, Strong L, et al. Expanding the clinical phenotype of hereditary BAP1 cancer predisposition syndrome, reporting three new cases. Genes Chromosomes Cancer. 2014;53:177-82. [PMCID: PMC4041196] [PubMed: 24243779]

25. Servois V, Mariani P, Malhaire C, Petras S, Piperno-Neumann S, Plancher C, et al. Preoperative staging of liver metastases from uveal melanoma by magnetic resonance imaging (MRI) and fluorodeoxyglucose-positron emission tomography (FDG-PET) Eur J Surg Oncol. 2010;36:189-94. [PubMed: 19775851]

Figures and Tables

Figure 1 


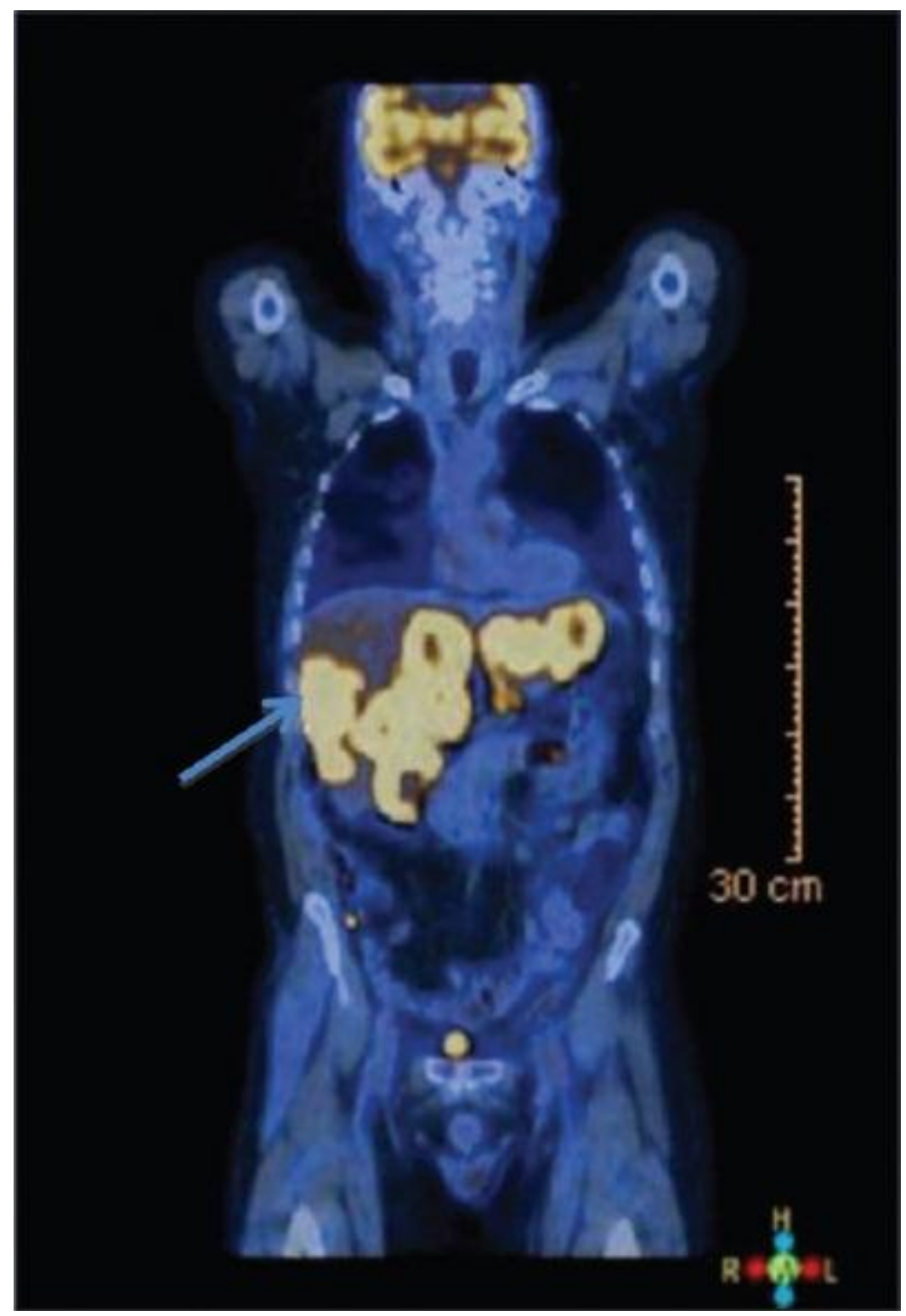

A coronal plane whole body (18) fluorodeoxyglucose positron emission tomography/computed tomography image. The highlighted arrow depicts extensive uptake in the liver consistent with a large metastatic tumour burden

\section{Table 1}

Patient demographics and tumor data 


\begin{tabular}{|c|c|}
\hline Tumour characteristics & Numbers \\
\hline Total number of patients & 108 \\
\hline Male (\%) & $50 / 108(46.3)$ \\
\hline Female $(\%)$ & $58 / 108(53.7)$ \\
\hline \multicolumn{2}{|c|}{ Tumor dimensions, median (mean $\pm S D$ ) } \\
\hline Tumor thickness (mm) & $6.5(5.2 \pm 1.8)$ \\
\hline Maximal base diameter $(\mathrm{mm})$ & $14.8(11.6 \pm 4.5)$ \\
\hline \multicolumn{2}{|l|}{ GOMS classification (\%) } \\
\hline Small melanomas & $5 / 108(4.6)$ \\
\hline Medium melanomas & $46 / 108(42.6)$ \\
\hline Large melanomas & $57 / 108(52.7)$ \\
\hline \multicolumn{2}{|l|}{ AJCC classification (\%) } \\
\hline I (T1NOMO) & $7 / 108(6.5)$ \\
\hline IA (T1aNOMO) & $2 / 108(4.8)$ \\
\hline IB (T1bNOMO) & $3 / 108(2.8)$ \\
\hline II (T2NOMO) & $12 / 108(11.1)$ \\
\hline III (T3NOMO) & $81 / 108(75)$ \\
\hline IVA (T4NOMO) & $0 / 108(0)$ \\
\hline IVB (T4T1M1) & $3 / 108(2.8)$ \\
\hline \multicolumn{2}{|l|}{ Tumor location (\%) } \\
\hline Choroidal & $83 / 108(76.8)$ \\
\hline Giliochoroidal & $15 / 108(13.8)$ \\
\hline Ciliary body & $7 / 108(6.5)$ \\
\hline Iridociliary & $1 / 108(0.9)$ \\
\hline Iris & $2 / 108(4.8)$ \\
\hline
\end{tabular}

COMS: Collaborative Ocular Melanoma Study, AJCC: American Joint

Committee on Cancer, SD: Standard deviation

Figure 2 


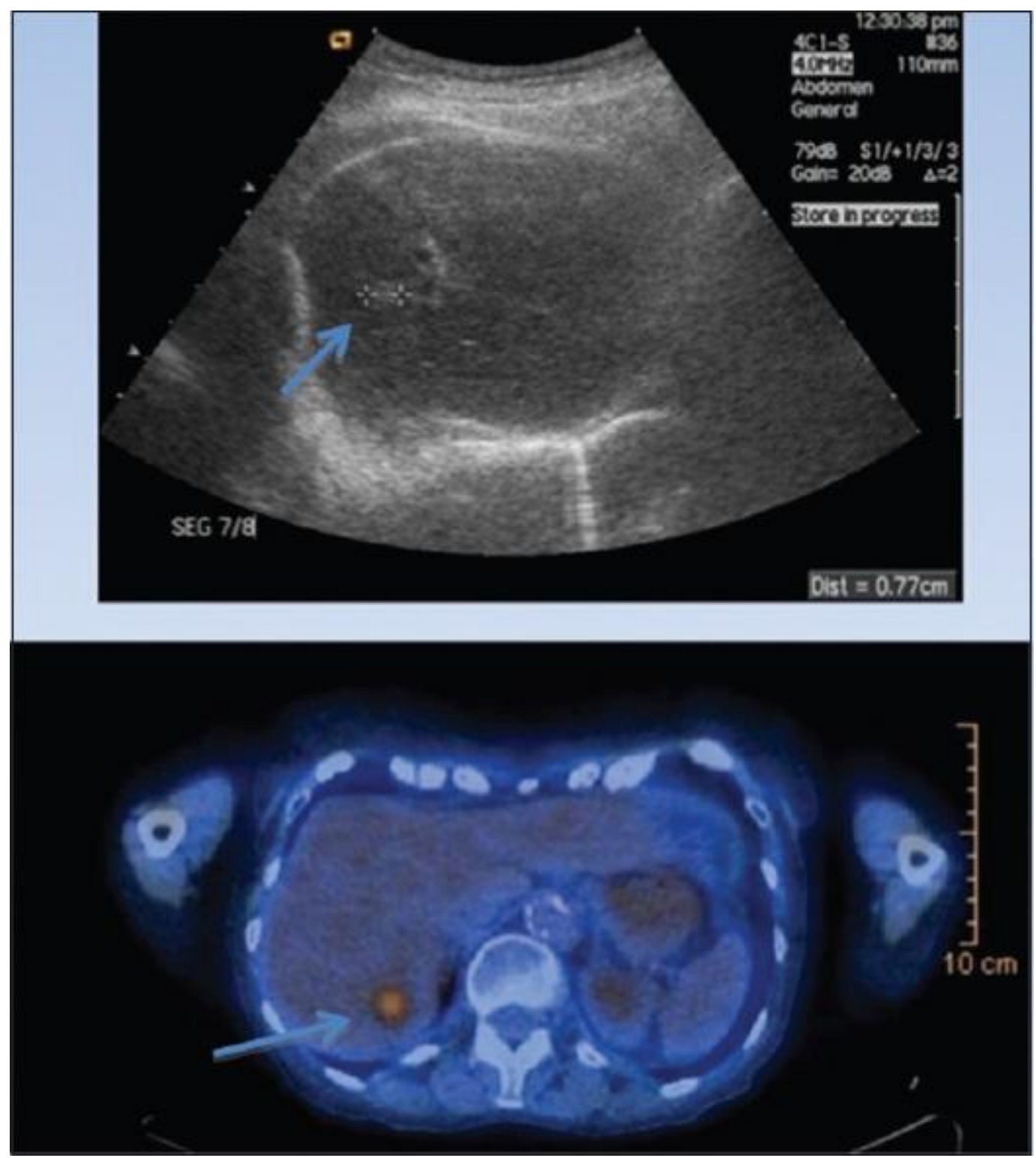

Comparison of imaging results for the same patient using liver ultrasound scan and a transverse plane (18) fluorodeoxyglucose positron emission tomography/computed tomography. The arrow indicates the location of a small isolated liver metastasis on ultrasound, which demonstrates fluorodeoxyglucose uptake on positron emission tomography/computed tomography 